\title{
Optimization of Central Pattern Generator based Quadruped Animation using Time Series with Genetic Algorithm
}

\begin{abstract}
Zeeshan Bhatti ${ }^{1}$, Shahneela Pitafi ${ }^{1}$, Naila Shabbir ${ }^{1}$
Abstract:

Generating believable quadruped motion is a challenging task for an application like Games, Virtual Reality, and Augmented Reality, where runtime user interaction is needed. In order to successfully generate the believable motion, the runtime or dynamic adjustment to motion gaits is essential. As the use of the Central Pattern Generator (CPG) is vital in generating a range of dynamic quadruped motions, the optimization is crucial for smooth motion curves. We use the Genetic Algorithm (GA) based tuning technique to optimize the motion curves generated through CPG. The quadruped motion generated through CPG is applied on Skeletal joints and then optimized through fitting the artificial motion parameters tuned using a genetic algorithm. The results generated show much smooth and stable quadruped motion with believable gait patterns.
\end{abstract}

Keywords: Central Pattern Generator, Quadruped Animation, Time Series, Genetic Algorithm

\section{Introduction}

Animation means giving motion to virtual objects created and manipulated using computers. The use of animation tools and techniques in modern times has immensely increased from application areas including TV, Movies, CGI, Games, Cartoons, VFX, Virtual and Augmented reality. With demand comes to the great responsibility of producing realistic or believable motions of virtual objects. The Quadruped animation has always posed a great challenge due to the increased number of articulated legs and a wide range of motion gait patterns[1][2].

The neurological analysis of quadruped motions has given the concept of the Central Pattern Generator (CPG) system, responsible for generating multiple locomotions and their transitions within each gait. The central pattern generator is a system that consists of a network of neurons residing inside the nervous system of multilegged animals. Along with many responsibilities, it is primarily responsible for maintaining accurate coordination between multiple legs during cyclic motion of multiple gaits and patterns [3]. The use of CPG to generate an animation of quadrupeds in a virtual environment is a very tricky task. In this research work, the motion generated from CPG is optimized to produce accurate believable motion curves using a Genetic Algorithm. The genetic algorithm is a very popular and common algorithm used commonly for heuristic search and optimization purposes in various fields.

\section{A. Central Pattern Generator for Motion}

Central Pattern Generator is a neurological circuit that is responsible for generating cyclic rhythmic motion patterns through neural activity. These neural circuits behave like a distributed system that consists of nonlinear coupled oscillators. The rhythmic motion signals are produced using the phase coupled oscillator principle

${ }^{1}$ Institute of Information and Communication Technology, University of Sindh, Jamshoro Corresponding Author: Zeeshan.bhatti@usindh.edu.pk 

with Genetic Algorithm

having different phase shifts for varying gait patterns. The change in gait patterns is achieved using altering the phase of coupling methods of oscillators[3].

In this research, the Phase oscillator is used as modelled by Kuramoto's [4]. The oscillator model consists of $\mathrm{N}$ coupled phase oscillators, where the phase difference of sine function is used to couple the phase oscillators. This principle is model mathematically as [5][6].

$$
\begin{aligned}
\dot{\theta}_{i} & =w_{i}+\sum_{j=1}^{N} \lambda_{i j} \sin \left(\theta_{j}-\theta_{i}-\Delta \phi_{i j}\right) \\
\ddot{r}_{i} & =\mu_{i}^{2}\left(R_{i}-r_{i}\right)-\frac{3}{2} \mu_{i} \dot{r}_{i} \\
x_{i} & =r_{i}\left[1+\sin \left(\theta_{i}\right)\right]
\end{aligned}
$$

Where phase of the $i^{\text {th }}$ oscillator is denoted by $\hat{\mathrm{I}}_{s i}$ and frequency by $\mathrm{I} \% o_{i}$. with its own frequency, the oscillator runs independently, while $\hat{I}_{{ }}{ }_{i j}$ is synchronizes it with all other oscillators. The phase shift parameter is $\Delta \hat{\mathrm{a}}^{\wedge} \cdots i j$ between the two oscillators $i$ and $j$. This type of CPG system consists of behaviors based on a stable limit cycle, due to which their output is modified by explicit parameters [7]. Figure 1 , shows the frequency and amplitude modulated based on the above principle.

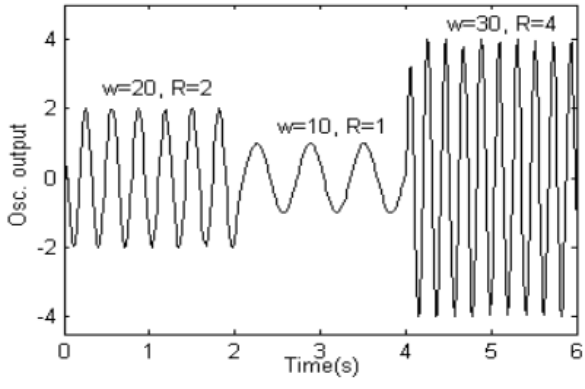

Fig 1: Change in frequency and amplitude of the oscillator (Source: [6])

\section{B. Genetic Algorithm}

Genetic algorithm is an optimization technique, belonging to a progressive class of artificial intelligence. The genetic algorithm randomly generates an initial population, then selects among the whole population the fittest by using the fitness function crossover between selected chromosomes and finally performs mutation and repeat cycle till the condition of the stop is true, as shown in generation cycle figure 2. A genetic algorithm consists of biological ideas like the population of chromosomes child production with cross over and mutation.

A Genetic algorithm is comparatively different than other commonly used optimization methods, as it is based on selecting the fittest from a given population. Genetic Algorithm does not use decision variables, it uses codes instead. For this type of optimization technique, binary or symbolic coding is necessary moreover genetic algorithm is a random process [8].

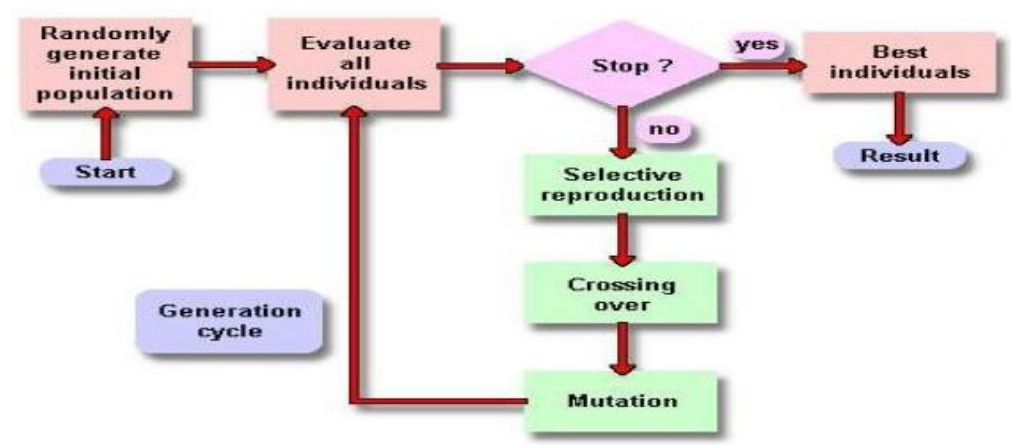

Fig 2: Genetic Algorithm presenting generation cycle (source: https://becominghuman.ai)

Sukkur IBA Journal of Computing and Mathematical Science - SJCMS | Vol. 4 No. 2 July - December 2020 () Sukkur IBA University 


\section{Literature Review}

A.P. Gerdelan et all in 2009 [9] proposed a Genetic-Fuzzy System (GFS) which is used for the optimization of animated character motion authors worked on a new thing that is an optimization of motion during running, this Genetic- Fuzzy system is ideal for gaming and simulation based on the real-time environment. Author has given a study to solve one of the drawbacks of fuzzy systems which is for every new type of character time-intensive manual calibration of system parameter is required. Similarly, Zühtü Hakan Akpolat et all in 2017 [10] used the Lagrange method and simulation of the robotic fish model in MATLAB/SimMechanics environment to produce the dynamic model of one active joint robotic fish. Langrange energy equation is used to drive the mathematical model of the system for the robotic fish inspired from a real carangiform fish and solidWorks is used to design the computeraided design (CAD) model of the robotic fish which is then transferred to simMechanics environment, results found for joint angles from SimMechanics and dynamic models are compared and proved with animation video of the robotic fish

Hiroshi Kimura et. al in [11] proposed a quadruped walk in a robot using a neural system model based on Central Pattern Generator (CPG) derived reflexes and responses. Response regulates the $\mathrm{CPG}$ phase, and reflex produces joint torque. Whereas, Katsuyoshi Tsujita et all in [12] proposed a control system for the fourlegged robot using nonlinear oscillators. The control system is based on a gait pattern that encompasses a nonlinear oscillator and a leg motion controller uses a local feedback control that initiates the actuators of the four legs. Similarly, Chengju LIU et. al in [13] also proposed a Central Pattern Generator (CPG) for a quadruped robot with reflex vestibular to produce a rhythmic motion. They used a function called kneeto-hip mapping and oscillator network to understand the elementary animal-like walking gait. Moreover, Chengju Liu et. al in (2009) [6] also generated different gaits and gait transitions by using CPG control system for monitoring the locomotion of a 4-legged robot.

Primarily, four basic quadruped gaits were produced using phase-locked oscillation shapes with mutually coupled phase oscillators built a body CPG network.

Abdalftah Elbori et all in (2018) [6] uses a couple of CPGs such as unidirectional, uncoupled, bidirectional. Abdalftah Elbori tries to apply hybrid function in genetic algorithms and debates on the stability analysis of CPGs moreover utilized the bidirectional two CPGs to familiarizes the CPGs to robotic systems that accomplish one-leg movement the results show that CPGs rheostat locomotion without any sensory feedback and increase movement as well

Whereas, Shivendra Shivani et all in [14] discussed the genetic algorithm with animation of the physical body to hit a target intelligently within an obstructed path. The genetic algorithm was used to train the objects and physical animation of the body for finding the accurate path and to be able to respond to the obstacles as the body collides with them.

L. GRITZ et. al proposed an AI-based Genetic Programming (GP) technique for achieving the goals of automatically derive the control program agents, his work [15]. It is a kind of motion prerequisite which is an alternative to key framing that permits a knowledge-based technique for highly automated generation of motion. L. GRITZ suggested that this method is a generalized approach as it can successfully generate the motion which is aesthetically tempting, specific to nature, and physically plausible.

J. J. Collins et all present in his work [17] a CPG model which is hard wired with nonlinear oscillators to generate numerous 3 specific gaits for quadruped including walk, trot \& bound motions, using oscillation patterns that are phase-locked. This was achieved by altering the oscillator 
parameters of the network's driving signal that yielded transitions between the different gaits are generated. Similarly, Bhatti, Zeeshan (2019) [18] discussed and developed a procedural animation framework that uses the same Central Pattern Generator (CPG) model to generate a coupled oscillation pattern for various quadruped characters, that produced oscillating in-phase and out-phase motion curves driven through kinematic joint chains and procedural programming techniques.

Bruno Floriano et all [19] proposed the use of a multi-objective Genetic Algorithm for generating optimized quadruped walk gait patterns with a balanced control using kinematics technique and empirical methodology. In order to maintain the stability of a quadruped robot with a consistent response, a balance control mechanism is developed proportionally with speed achieved through measurements of the embedded accelerometer. Whereas, R. Singh ET all proposed [16] a model for locomotion of quadruped robot with the application of obstacle avoidance based on the fuzzy logic controller. This controller detects and implements the obstacle avoidance mechanism using three main Ultrasonic sensors (HC-SR04) mounted at the front of the robot. Results show the obstacle avoidance fuzzy controller and navigation work successfully.

\section{Quadruped Model:}

The quadruped model and skeletal structure used in this work are of a Lion and Horse character [20]. Each leg consists of 6 joints with joints near hips and front shoulders having a ball joint with 2 degrees of freedom, whereas, the knee and ankle joints consist of ball joints with 1 degree of freedom [21], as shown in Figure 3(a) and (b). In this research, the motion of the spine and neck have not been considered and calculated to limit the focus work and so are not discussed further.

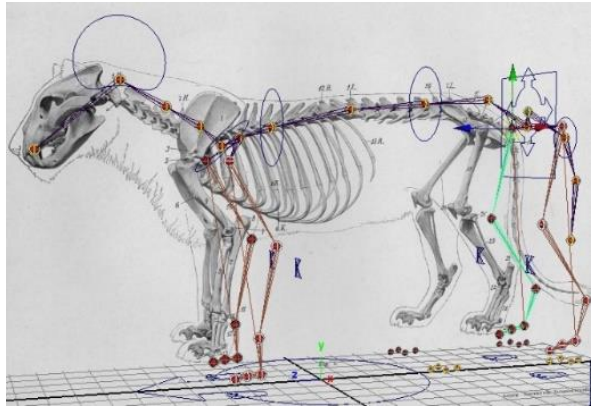

Fig 3(a): Skeletal joint structure of Lion

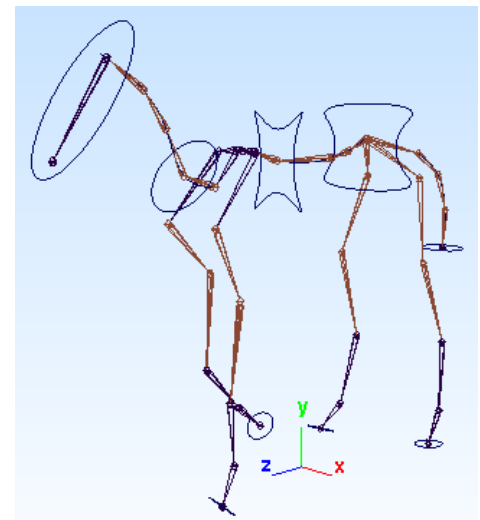

Fig 3(b): Skeletal Joint structure of Horse

\section{Central Pattern Generator (CPG) for Quadruped}

The animal gaits possess a type of permutation symmetry which is grouped and characterized according to its type. Based on this, each leg of a multi-legged animal is assumed to possess a subnetwork of neurons driven through individual and separate CPG for each leg. Most of the CPGs work on this principle, with interlimb synchronization and coordination achieved through coupling these multiple CPG networks together. According to this, the system implements multiple units of CPG's based on the coupled oscillator principle.

This system then directly controls each leg's motion and generate a coupled pattern of symmetrical rhythmic oscillation. By using these multiple units of CPGs on each leg, the coupling of leg In-a-Phase, leg Outof-Phase, and variable phases are done, generating different rhythmic behaviors. 

with Genetic Algorithm

These varying rhythmic behaviors include gait patterns of a different family of animals, i.e, Lion tiger, Horse, with walking, trotting, galloping, and others [22][18].

\section{A. Systems of Two Coupled Oscillators}

A coupled phase oscillator model is used in our quadruped CPG system to modulate the sinusoidal patterns of leg motion as discussed in [23]. The model of coupled phase oscillator allows us to synchronize the various periodic gait patterns of quadrupeds, generated through the CPG. In a typical system, by coupling two identical oscillators, two oscillation patterns are obtained as a result [24] [3]

The In-Phase pattern: in this technique the waveforms generated is the same from both the oscillators

The Out-of-Phase pattern: in this technique the waveforms generated are the same, however, there is a difference of half a period between each wave obtained from both the oscillators.

The motion analysis study shows that the gaits of quadruped animals move with a periodic cyclic pattern in a symmetrical rhythm, as the four legs are already located in symmetry [25].

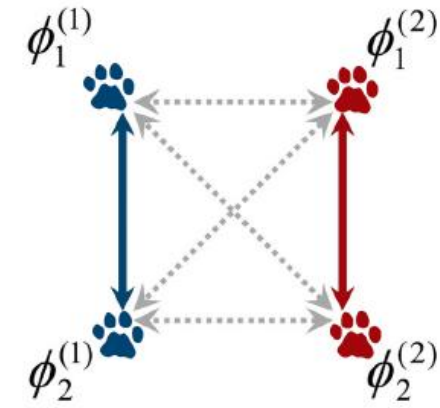

Fig 4: The Lateral and Diagonal couplings are represented by the solid and dotted lines, respectively for a quadruped gait.

The symmetrical coupling of the four legs is represented in Figure (3), where solid bars show the lateral and dotted lines show the diagonal synchronization. A cycle is represented by the interval between successive foot strikes of the same foot, from where the sequence is repeated [3]. The foot strike factor, determined by impact phase (dip), is the fraction of a cycle when the foot is in contact with the ground. The motion of quadruped phenotypes produces two variants of gait phases, where the legs are out-of-phase (walking or running) or inphase (jumping or hopping), with some gaits having a more complex behavior of the relative phase [26][3][21].

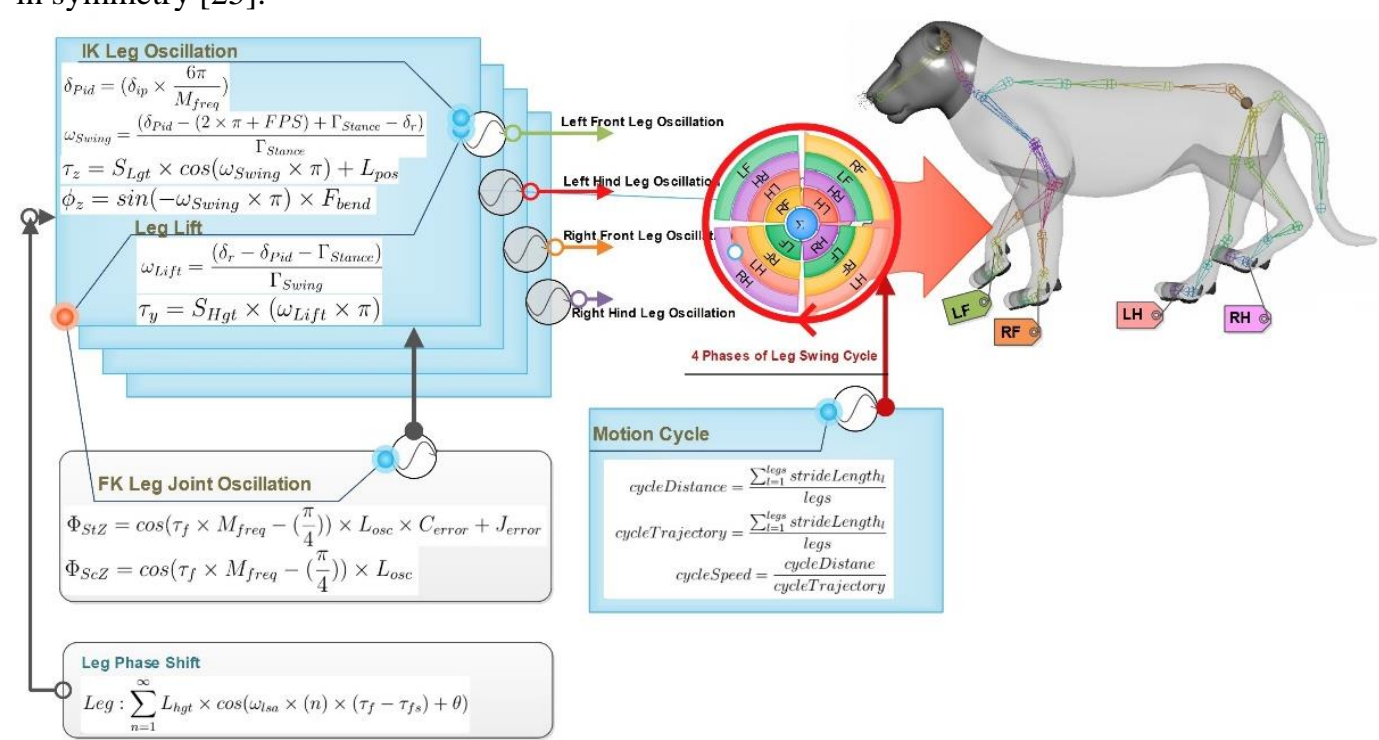

Fig 5: Mathematical model for CPG based Leg Simulation 


\section{Optimization Model for Motion Curves}

To optimize the animation curve, a Time series problem is applied to motion curves generated for quadruped animation. The time series can be applied on various data sets that change with respect to times, to determine and predict complex, nonperiodic, irregular, and chaotic sequential data that vary with respect to time [27]. In order to optimize the animation of the quadruped, the motion curve is considered as Time Series, and the Genetic algorithm is applied to the keyframe data.

\section{A. Optimization with Time Series}

Animation Motion curve data in a time series (T) can be formulated as $T=\left\{x_{t}\right.$, $t=1, \ldots, N\}$, where $t$ is known as the time in frames, $\mathrm{N}$ is the total number of Frames. Since, typically a time series is defined as "a sequence of observed data, usually ordered in time" [28], the proposed framework can be used with a Genetic algorithm for curve optimization and prediction of next motion curve values, generated by the Central Pattern Generator (CPG). The problem of removing noise from a motion curve generated procedurally or through motion capture data can be better understood from figure 4 . The diamond shape point represents the keyframe generated automatically by CPG, which can be seen as to be noisy, irregular, and nonperiodic, resulting in non-smooth animation. Whereas, the square boxes indicate the key poses that may be considered as the most important and required frames. Now, here the goal is to predict and optimize these motion curves as accurately as possible.

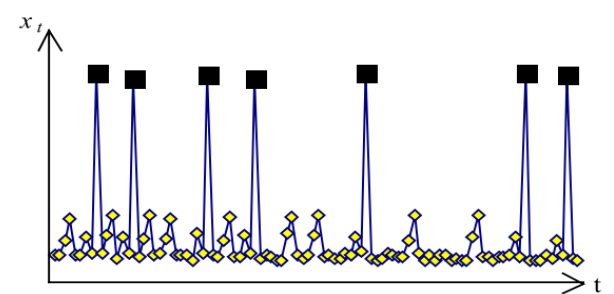

Fig 4: Time series values ([27]).
In a time, series, an event is considered important, whereas in our model the event is known as Key Pose. Within a time, series, temporal pattern, and keyframe characterization function are adopted as the fundamentals. Here, the temporal pattern is considered as unknown keyframe data that is characterized and used for the prediction of possible accurate key poses within a motion series.

The formula for the temporal pattern is obtained from [27], where a cluster of the temporal pattern (p) is defined as the set of all points within $\delta$ and it's a vector of length $Q$.

$$
P=\left\{a \in \mathbb{R}^{Q}: d(\mathbf{p}, a) \leq \delta\right\}
$$

where, the distance between frames is d, with $Q$ defined as hypersphere of dimension, $\delta$ is the radius, $\mathbf{p}$ here is center. The $\mathbf{p} \in \mathbb{R}^{Q}$ is the representation of a point $Q$ dimensional real metric space of a temporal pattern [27].

\section{B. Optimization with Genetic Algorithm}

Genetic Algorithm is used and adapted to the characterization of Motion curve with time-series framework. The typical implementation model of a genetic algorithm for optimization is described as

- The population of key poses and keyframes with respect to time in frames, that are evaluated with fitness function.

- Generation of a large population of random key poses, based on a genetic operation involving the selection of next generations, crossover mechanism, mutation of key poses, and tuning of keyframes.

- Determines the fitness by using a gradient search method to improve convergence performance [29].

- $\quad$ Select the top $1 / \mathrm{n}$ of the top population to begin

- While all fitness has not met

- Next Generation Selection

- Crossover

- Mutation

- Tuning 


\section{Selection of the Next Generation}

The core structure of GA is based on the survivor of the fittest principle. According to this principle, certain key poses and their keyframes will be selected from the population, based on their fitness scores. This produces the foundation for the next generation resulting in producing individual offspring knows as parents. These parents are randomly selected based on the fittest individuals. This leads to a crossover ratio, in which the best parents are obtained. Then the mutation of key poses is done on randomly selected parents.

\section{The crossover Mechanism}

Crossover typically is a key stage in GA which works at chromosome level and splits the string of chromosomes at a random position. This produces two individual segments of each chromosome knowns as heads and tails as shown in figure 5 .

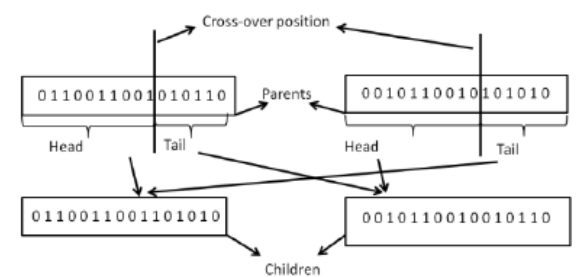

Fig 5: A sample crossover

\section{The Mutation Mechanism}

Mutation comes after the crossover process, which alters the gene of randomly chosen individuals key poses on a time frame. This provides us with the mechanism to random search each small keyframe with a small amount and ensures that all in-between frames with small pose change are searched with zero probability of missing the search space as illustrated in Figure 6.

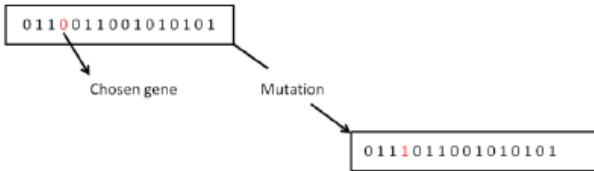

Fig 6: Mutation Scheme

\section{The Tuning process.}

The final tuning of motion curves was achieved by defining complete parameters and individual keyframes and their corresponding key poses with a given set of the population as shown in the figure. The total amount of cross-over population was at $55 \%$, with the number of individual mutations at $16 \%$. The elite individual keyframe for the animation within 30 frames per second (fps), was also around $16 \%$, as discussed in [8]. The total number of populations of keyframes opted for crossover and mutation were initially parameterized with the selected elite parents of the population.

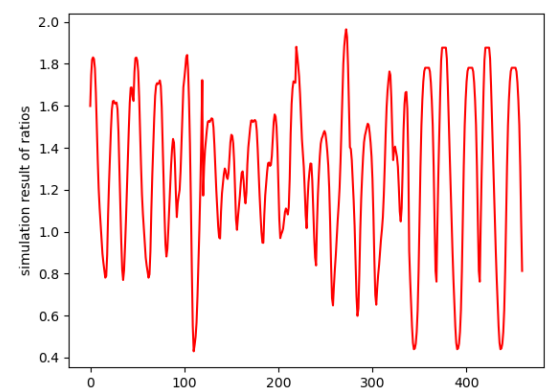

Fig 7: Motion curve of front leg joint

The Optimization model used based on genetic algorithm is illustrated in Figure 8. This model was applied on a quadruped model with 18 main joints of all four legs. The leg locomotion is automated through CPG as discussed in [18] with the biomechanics of joints having multiple degrees of freedom of joints already discussed in [21].

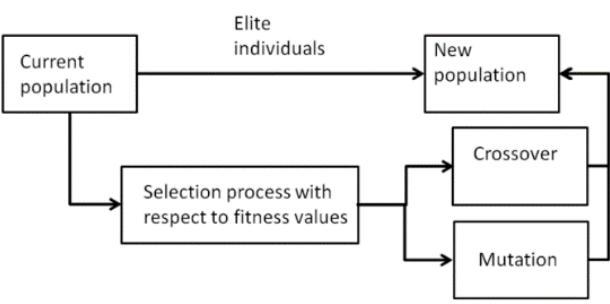

Fig 8: Genetic Algorithm based optimization scheme (source: [8])

This prototype model was used with only walk motion achieving curves at different 
Zeeshan Bhatti (et al.), Optimization of Central Pattern Generator based Quadruped Animation using Time Series with Genetic Algorithm

joints and optimized using the time series parameters with a genetic algorithm. Figure 9 (a-d) shows the final motion curves of

each joint of quadruped while in walk motion.

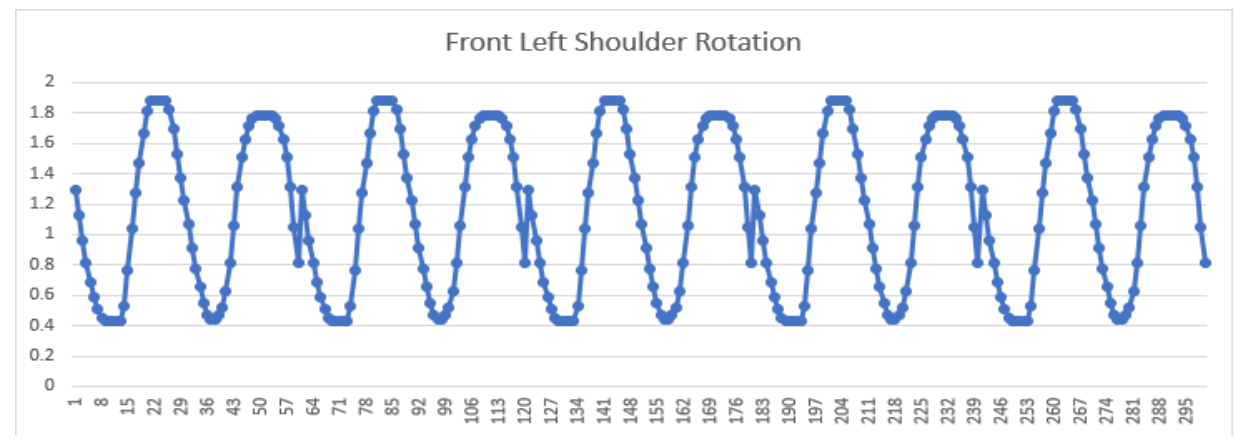

Fig 9(a): Motion curve of Front Left Shoulder Joint of a tiger during the walk

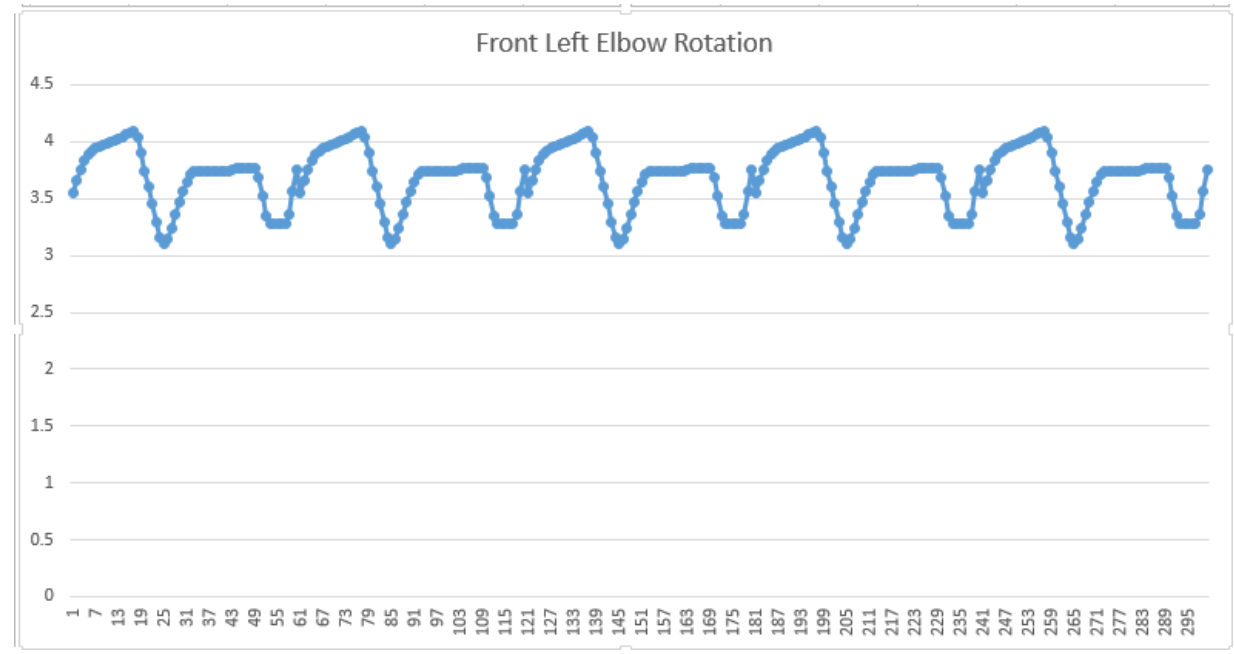

Fig 9(b): Motion curve of Front Left Elbow Joint of a tiger during the walk

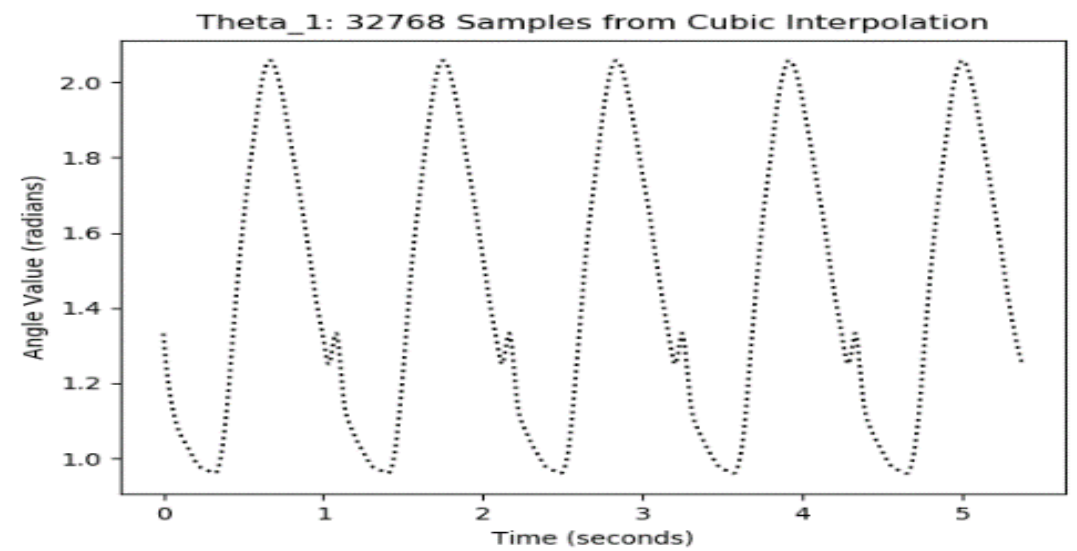

Fig 9(c): Optimized Motion curve of Right Shoulder Joint of a tiger during the walk

Sukkur IBA Journal of Computing and Mathematical Science - SJCMS | Vol. 4 No. 2 July - December 2020 @ Sukkur IBA University 


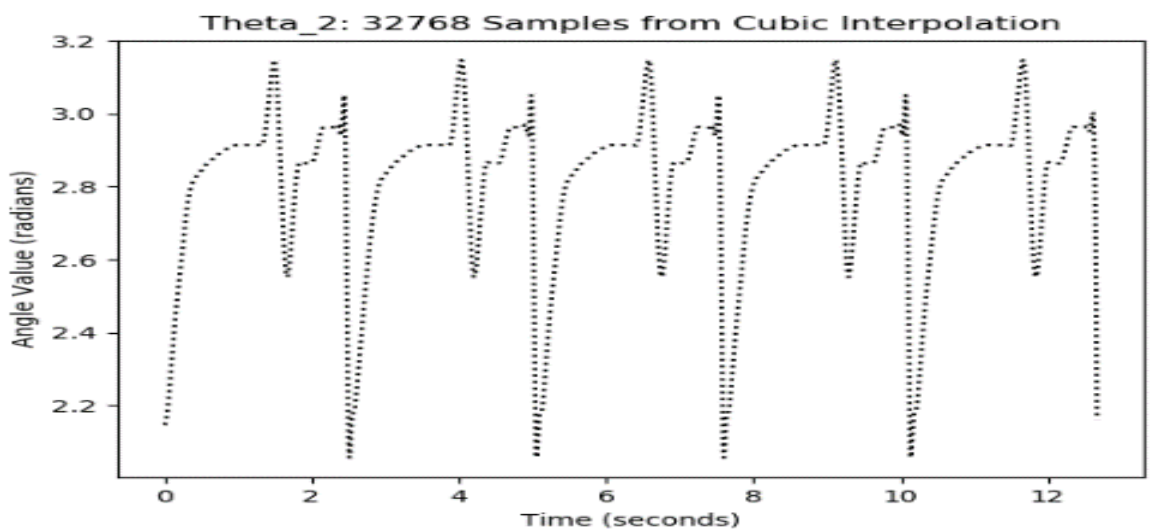

Fig 9(d): Optimized Motion curve of Right Elbow Joint of a tiger during the walk

\section{Conclusion}

In this paper, an optimization model is discussed using a genetic algorithm with the time series to correct and optimize the motion curve of a quadruped generated through Central Pattern Generator (CPG). CPG is a neurological mechanism, used by quadruped to generate various different locomotion's. While, this is fairly complex, generating accurate and believable motion curves based on Oscillating CPG is a difficult task. The use of an optimization technique based on a genetic algorithm, while characterizing the motion curve with respect to time series, generated a very believable animation and reduced the noise and anomalies in animation. The results showed to be very promising with the animation of each joint looking more natural and believable.

\section{Reference}

[1] Z. Bhatti, A. Shah, A. Waqas, and M. Karbasi, "Automated animation of quadrupeds using procedural programming technique," Asian $J$. Sci. Res., vol. 8, no. 2, pp. 165-181, 2015.

[2] Z. Bhatti, A. Shah, and F. Shahidi, "Procedural Model of Horse Simulation," in 12th ACM SIGGRAPH International Conference on Virtual-Reality Continuum and Its Applications in Industry (ACMSIGGRAPH VRCAI 2013), 2013, pp.
139-146.

[3] H. Castellini, E. Yudiarsah, L. Romanelli, and H. A. Cerdeira, "Coupled chaotic oscillators and their relation to a central pattern generator for artificial quadrupeds," Pramana J. Phys., vol. 64, no. 4, pp. 525-534, 2005.

[4] J. A. Acebrón, L. L. Bonilla, C. J. P. Vicente, F. Ritort, and R. Spigler, "The Kuramoto model: A simple paradigm for synchronization phenomena," Rev. Mod. Phys., vol. 77, no. 1, pp. 137-185, 2005.

[5] Ö. K. Adak, O. Ayit, M. Gülhan, and K. Erbatur, "Genetic Tuning of a Central Pattern Generator for Quadruped Locomotion," in In 3rd International Electrical and Electronic Engineering and Technologies Conference (ELECTROTECH 2015)., 2015, no. October.

[6] C. Liu, Y. Chen, J. Zhang, and Q. Chen, "CPG driven locomotion control of quadruped robot," Conf. Proc. - IEEE Int. Conf. Syst. Man Cybern., no. 60875057, pp. 23682373, 2009.

[7] ÖMER KEMAL ADAK, "QUADRUPED LOCOMOTION REFERENCE SYNTHESIS WITH CENTRAL PATTERN GENERATORS TUNED BY EVOLUTIONARY

ALGORITHMS," Sabanci 
[8] Ö. K. Adak, O. Ayit, M. Gülhan, and K. Erbatur, "Genetic Tuning of a Central Pattern Generator for Quadruped Locomotion," 3rd Int. Electr. Electron. Eng. Technol. Conf. (ELECTROTECH 2015)., no. October, 2015.

[9] A. P. Gerdelan, "Optimising Animated Character Motion with a New Fuzzy-Genetic Hybrid Method: Fuzzy Controllers in Ani- mation," 2009.

[10] Z. H. Akpolat, M. Bingöl, M. Ay, G. Özmen Koca, C. Bal, and D. Korkmaz, "Dynamic Model and Simulation of One Active Joint Robotic Fish," e-Journal New World Sci. Acad., vol. 12, no. 1, pp. 29-39, 2017.

[11] H. Kimura, Y. Fukuoka, and A. H. Cohen, "Adaptive dynamic walking of a quadruped robot on natural ground based on biological concepts," Int. J. Rob. Res., vol. 26, no. 5, pp. 475-490, 2007.

[12] K. Tsujita, K. Tsuchiya, and A. Onat, "Adaptive gait pattern control of a quadruped locomotion robot," IEEE Int. Conf. Intell. Robot. Syst., vol. 4, pp. 2318-2325, 2001.

[13] C. Liu, Q. Chen, and G. Wang, "Adaptive walking control of quadruped robots based on central pattern generator (CPG) and reflex," J. Control Theory Appl., vol. 11, no. 3, pp. 386-392, 2013.

[14] S. Shivani and S. Tiwari, "Simulation of intelligent target hitting in obstructed path using physical body animation and genetic algorithm," Multimed. Tools Appl., vol. 78, no. 8, pp. 9763-9790, 2019.

[15] L. Gritz and J. K. Hahn, "Genetic programming for articulated figure motion," J. Vis. Comput. Animat., vol. 6, no. 3, pp. 129-142, 1995

[16] T. K. Singh, R., \& Bera, "Walking Model of Jansen Mechanism-Based Quadruped Robot and Application to Obstacle Avoidance," Arab. J. Sci. Eng., vol. 45, no. 2, pp. 653-664, 2020 .
[17] J. J. Collins and S. A. Richmond, "Hard-wired central pattern generators for quadrupedal locomotion," Biol. Cybern., vol. 71, no. 5, pp. 375-385, 1994.

[18] Z. Bhatti, "Oscillator driven central pattern generator (CPG) system for procedural animation of quadruped locomotion," Multimed. Tools Appl., vol. 78, no. 21, pp. 30485-30502, 2019.

[19] A. R. S. de Oliveira Floriano, B. R., de Freitas Porphirio, C., Santana, P. H. M., Borges, G. A., \& Romariz, "Walking pattern design and balance control of a quadruped platform," in Latin American Robotic Symposium, 2018 Brazilian Symposium on Robotics (SBR) and 2018 Workshop on Robotics in Education (WRE), 2018, pp. 242-247.

[20] Z. Bhati, A. Shah, A. Waqas, H. Abid, and M. Malik, "Template based Procedural Rigging of Quadrupeds with Custom Manipulators," in International Conference on Advanced Computer Science Applications and Technologies, 2013, pp. 259-264.

[21] Z. Bhatti, A. Waqas, A. W. Mahesar, and M. Karbasi, "Gait Analysis and Biomechanics of Quadruped Motion for procedural Animation and Robotic Simulation," Bahria Univ. J. Inf. Commun. Technol., vol. 10, no. 2, pp. 1-7, 2017.

[22] F. Lacquaniti, R. Grasso, and M. Zago, "Motor Patterns in Walking," News Physiol. Sci., vol. 14, no. August, 1999.

[23] J. Morimoto, S. Hyon, G. Cheng, D. Bentivegna, and C. G. Atkeson, "Modulation of simple sinusoidal patterns by a coupled oscillator model for biped walking," in Proceedings 2006 IEEE International Conference on Robotics and Automation, 2006. ICRA 2006., 2006, pp. 1579-1584.

[24] Y. Kawamura, "Phase synchronization between collective rhythms of fully locked oscillator groups.," Sci. Rep., vol. 4, no. C, p. 
4832, Jan. 2014.

[25] Z. Bhatti, A. Shah, M. Karabasi, and W. Mahesar, "Expression driven Trignometric based Procedural Animation of Quadrupeds," in Informatics and Creative Multimedia (ICICM), $2013 \quad$ International Conference on, pp. 104-109.

[26] R. A., Principles of Locomotion. Princeton, New Jersey: Princeton University Press, 2003.

[27] R. J. Povinelli, "Using Genetic Algorithms To Find Temporal
Patterns Indicative Of," GECCO 2000 Work. Data Min. with Evol. Algorithms, no. March, pp. 80-84, 2000.

[28] S. M. Pandit and S.-M. Wu, Time series and system analysis, with applications. New York: Wiley, 1983.

[29] J. H. Lee and J. H. Park, "Timedependent genetic algorithm and its application to quadruped's locomotion," Rob. Auton. Syst., vol. 112, pp. 60-71, 2019. 